\title{
A multicentre evaluation of emergency abdominal surgery in South Africa: Results from the GlobalSurg-1 South Africa study
}

\author{
R T Spence, ${ }^{1,2}$ MB ChB, MPhil; E Panieri, ${ }^{2}$ MB ChB, FCS (SA); S L Rayne, ${ }^{3}$ MB ChB, FCS (SA); on behalf of the GlobalSurg \\ South Africa collaboration \\ ${ }^{1}$ Codman Center, Department of General Surgery, Massachusetts General Hospital, Boston, USA \\ ${ }^{2}$ Department of Surgery, Faculty of Health Sciences, University of Cape Town, South Africa \\ ${ }^{3}$ Department of Surgery, Faculty of Health Sciences, University of the Witwatersrand, Johannesburg, South Africa
}

Corresponding author: $R$ T Spence (rtspence@mgh.harvard.edu)

Background. GlobalSurg-1 was a multicentre, international, prospective cohort study conducted to address the global lack of surgical outcomes data. Six South African (SA) hospitals participated in the landmark surgical outcomes study. In this subsequent study, we collated the data from these six local participants and hypothesised that the location of surgery was an independent risk factor for an adverse outcome following emergency intraperitoneal surgery.

Methods. Participating hospitals contributed 30-day outcomes data of consecutive emergency intraperitoneal surgical operations performed during a 2-week period between July and November 2014. The six heterogeneous hospital cohorts were compared by categorical confounders. The primary outcome measure was in-hospital mortality; secondary outcome measures were in-hospital morbidity and length of stay of $>14$ days. The unadjusted association between hospital and adverse outcome and the univariate association between categorical confounders and adverse outcome were tested. Significant associations were further tested by a multivariate stepwise forward logistic regression model built for each outcome of interest.

Results. Six hospitals (designated 1 - 6) contributed outcomes data for 169 operations. The mean age of the patients was 34.9 years (range 9 - 82), 116 (68.6\%) were male, and the majority (37.2\%) presented as a result of trauma. Hospital 5 was associated with 76 -fold increased odds of in-hospital death and 58-fold increased odds of a major in-hospital complication, and hospital 3 was associated with 3-fold increased odds of any in-hospital complication. The final model predicting in-hospital death had a receiver operating characteristic curve statistic of 0.8892 . Conclusion. The hospital is an independent risk factor for risk-adjusted adverse outcomes following emergency intraperitoneal surgery in SA.

S Afr Med J 2016;106(2):163-168. DOI:10.7196/SAMJ.2016.v106i2.10183

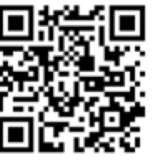

Two hundred and fifty million surgical operations are performed annually worldwide, and approximately 28 $32 \%$ of the global burden of disease is due to surgical reversible disease. Only $3.5 \%$ of these operations will be performed on the poorest third of the world's population. ${ }^{[1]}$ Global surgery is increasingly being recognised as a new priority in reducing global mortality. In high-income countries such as the UK, about $10 \%$ of patients undergoing surgery are at high risk of complications, and they account for $80 \%$ of postoperative deaths. ${ }^{[2]}$ This burden is likely to be much higher in low- and middle-income countries (LMICs), but clinical outcomes following major surgery are poorly described at national and even at institutional level in most countries, including South Africa (SA). ${ }^{[3]}$

The GlobalSurg collaborative has been a pioneering attempt to address this lack of surgical outcomes data through multicentre international prospective data collection. The investigators hypothesised that emergency abdominal surgery, including laparotomy, appendicectomy and hernia repair, is performed in surgical units throughout the world and is likely to be subject to performance variation. ${ }^{[4]}$ With no national database on non-cardiac

The GlobalSurg-1 South Africa collaboration: J-A Carreira, K Connor, V Jennings, H Kretzmann, D Nel, N Ngayu, A M Mphatsoe, R Moore, C Sampson, N Sishuba, E Teasdale, M Tun, M Wagener, E Harrison, J Fitzgerald, A Bhangu. surgical outcomes in the public or private sector in SA, the degree to which this variation exists here is not currently known.

\section{Objectives}

The primary objective of this study was to use data from SA hospitals that contributed to GlobalSurg-1 to compare risk-adjusted adverse outcomes among participating hospitals. We hypothesised that the location of surgery was an independent risk factor for an adverse outcome following emergency intraperitoneal surgery. The secondary objective was to contribute to the growing evidence that national benchmarking of surgical outcomes is both feasible and important, even in a resource-limited country.

\section{Methods}

\section{Patient population}

GlobalSurg-1 was a multicentre, prospective observational study of consecutive patients undergoing emergency intraperitoneal surgery. Any hospital in the world was eligible to enter, and the study was carried out during 14-day consecutive time periods of the individual participants' choice during a 5-month study period window between July and November 2014. Patients (adult and paediatric) of all ages undergoing emergency intraperitoneal surgery during the chosen period were eligible for inclusion. Emergency procedures were defined as unplanned, non-elective operations and included reoperations after previous procedures. Intraperitoneal surgery included laparoscopic, 
laparoscopic converted and open cases. This could have included gastrointestinal, vascular, urological and gynaecological surgery. Elective (planned) or semi-elective (where the patient is initially admitted as an emergency, then discharged from hospital and readmitted at a later time for surgery) procedures were excluded, along with caesarean sections. Centres had to ensure that they included consecutive patients and provided $>95 \%$ data completeness (centres with $>5 \%$ missing data were excluded from analysis). There was no minimum number of patients per centre.

Six SA hospitals that contributed to GlobalSurg-1 were included in the final analysis and made up the study population for this study. Three hospitals were academic teaching hospitals and three were secondarylevel institutions. All participating hospitals were in the public sector.

\section{Data collection}

In the original GlobalSurg-1 study, data points related to the patient, surgeon, operation, hospital, operative method and postoperative period were collected. The original data sheet can be accessed online (http://bmjopen.bmj.com/content/4/10/ e006239/T1.expansion.html). For this SA study, variables were categorised according to our new model for surgical outcomes research, where factors are separated into the domains of patient, presenting problem, provider, process of care and postoperative course. In this manner, the '5P' of surgical outcomes research has been piloted. Local investigators entered data via a secure online web page, provided using the Research Electronic Data Capture (REDCap) system hosted at the University of Edinburgh, Scotland. ${ }^{[5]}$ All patient data were transmitted and held anonymously. The data for GlobalSurg-1 were not analysed at identifiable hospital or surgeon level. In this study, the hospitals were anonymised before local data analysis.

\section{Classification of endpoints}

The primary endpoint for this study was in-hospital mortality. This was generated by combining the 'intraoperative mortality' and 'died as inpatient after surgery' variables collected in GlobalSurg-1. In-hospital mortality was chosen because it is a clear endpoint with a small chance of misclassification bias. Thirtyday mortality was reported inconsistently in the original study. Secondary endpoints included in-hospital complications (major, minor and any) and length of stay (LOS) greater than 14 days (LOS>14). A major in-hospital complication was defined as an intraoperative or postoperative complication that led to an unplanned 30-day critical care (intensive care unit) admission. Minor in-hospital complications were defined as an intraoperative or postoperative complication that did not lead to critical care admission. Any in-hospital complication represented the presence or absence of either an in-hospital death or a major or minor complication. LOS $>14$ was generated by dichotomising the length of stay into $>14$ days or $\leq 14$ days from the day of surgery.

\section{Analysis}

To explore the association between hospital site and the defined endpoints, the six participating hospitals were first compared by the spread of the categorical confounder variables included in our 5P framework. The unadjusted association between hospital and outcome was then tested. A univariate screen between all other categorical confounders and outcome was also performed. Pearson's $\chi^{2}$ test or Fisher's exact test where appropriate was used for both thesesteps. Any confounder that wasstatistically associated with the outcome at the 0.1 level was then entered into a stepwise forward entry logistic regression algorithm predicting the outcome of interest. The significance level for entry into the final logistic regression model was 0.05 . The final models predicting all five outcomes of interest are presented. The model's discriminatory ability to predict the primary binary outcome of in-hospital mortality was assessed by constructing the receiver operating characteristic (ROC) curve. ROC statistics range from 0 to 1,0 indicating no discriminatory ability and 1 perfect discriminatory ability.

\section{Ethical considerations}

Ethics approval was received from the appropriate SA university human research ethics committees for each participating hospital.

\section{Results}

\section{Patient characteristics}

The six SA hospitals participating in GlobalSurg-1 contributed 169 patients to the multicentre international evaluation and vividly represented the significant trauma burden our country faces relative to other participating countries (Fig. 1). Patient characteristics in each hospital are presented in Table 1, compared by categorical characteristics of the patients, providers, presenting problems and process of care received. The mean age of the patients undergoing surgery was 34.9 years (range 9 82); 116 were male (68.6\%) and 53 female (31.4). The greatest proportion of patients presented as a result of trauma $(n=61$, $37.2 \%)$, followed by acute appendicitis ( $n=41,24.4 \%)$, symptomatic ventral hernia $(n=12,7.1 \%)$ and perforated peptic ulcer $(n=10,6.0 \%)$. There was significant variation in hospital cohorts. Patient characteristics differed by gender $(p=0.016)$, American Association of Anesthesiologists (ASA) score $(p<0.001)$, prevalence of diabetes $(p=0.027)$ and prevalence of current smoking $(p=0.031)$. The presenting problem also differed markedly, with hospital 2 treating the greatest proportion of trauma (48.7\%) and hospital 6 treating the greatest proportion of acute appendicitis $(59.1 \%$; $p<0.0001)$.

The presence of specialist medical practitioners varied significantly among the hospitals $(p<0.0001)$. Specialist surgeons were present in only $10.8 \%$ of operations in hospital 2 but in $86.4 \%$ in hospital 6, while specialist anaesthetists were present in $5.6 \%$ in hospital 4 and $100 \%$ in hospital 2 . The process of care differed among the hospitals with reference to the preoperative delay to theatre $(p=0.003)$, use of preoperative computed tomography (CT) scanning $(p<0.0001)$, fashioning of any form of stoma $(p=0.038)$ and the operation

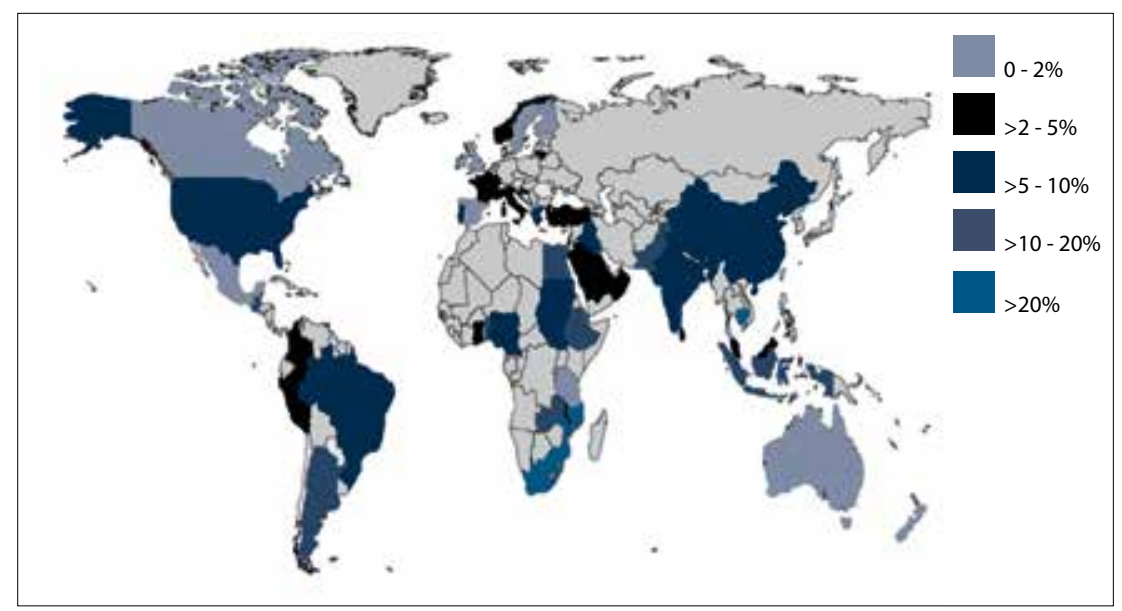

Fig. 1. Proportion of trauma as the presenting problem in GlobalSurg-1. (Reproduced with permission from the GlobalSurg-1 collaborators.) 
Table 1. Comparison of patient characteristics $(N=169)$ by participating hospital

\begin{tabular}{|c|c|c|c|c|c|c|c|}
\hline \multirow[b]{2}{*}{ Patient characteristics } & \multicolumn{6}{|c|}{ Hospital } & \multirow[b]{2}{*}{$p$-value } \\
\hline & 1 & 2 & 3 & 4 & 5 & 6 & \\
\hline \multicolumn{8}{|l|}{ Patient } \\
\hline Total patients, $n(\%)$ & $35(20.7)$ & $37(21.9)$ & $4727.8)$ & $18(10.7)$ & $10(5.9)$ & $22(13.0)$ & \\
\hline Age category, $n(\%)$ & & & & & & & 0.226 \\
\hline Paediatric & $4(11.4)$ & $3(8.1)$ & $1(2.1)$ & $2(11.1)$ & $1(10.0)$ & $5(22.7)$ & \\
\hline Adult & $29(82.9)$ & $33(89.2)$ & $43(91.5)$ & $13(72.2)$ & $9(90.0)$ & $16(72.7)$ & \\
\hline Elderly & $2(5.7)$ & $1(2.7)$ & $3(6.4)$ & $3(6.4)$ & $0(0.0)$ & $1(4.6)$ & \\
\hline Gender, $n(\%)$ & & & & & & & 0.016 \\
\hline Male & $27(77.1)$ & $23(62.2)$ & $30(63.8)$ & $8(44.4)$ & $10(100.0)$ & $18(81.8)$ & \\
\hline Female & $8(22.9)$ & $14(37.8)$ & $17(36.2)$ & $10(55.6)$ & $0(0.0)$ & $4(18.2)$ & \\
\hline ASA score & & & & & & & 0.0007 \\
\hline 1 & $26(74.2)$ & $12(32.1)$ & $19(45.2)$ & $9(50.0)$ & $10(100.0)$ & $18(81.8)$ & \\
\hline 2 & $6(17.1)$ & $12(32.1)$ & $8(19.1)$ & $3(16.7)$ & $0(0.0)$ & $2(9.1)$ & \\
\hline 3 & $1(2.9)$ & $8(21.6)$ & $9(21.4)$ & $4(22.2)$ & $0(0.0)$ & $2(9.1)$ & \\
\hline 4 & $2(5.7)$ & $3(8.1)$ & $6(14.3)$ & $1(5.6)$ & $0(0.0)$ & $0(0.0)$ & \\
\hline 5 & $0(0.0)$ & $2(5.4)$ & $0(0.0)$ & $1(5.6)$ & $0(0.0)$ & $0(0.0)$ & \\
\hline Diabetes, $n(\%)$ & & & & & & & 0.027 \\
\hline Present & $35(100.0)$ & $34(91.9)$ & $45(95.7)$ & $15(83.3)$ & $8(80.0)$ & $22(100.0)$ & \\
\hline Absent & $0(0.0)$ & $3(8.1)$ & $2(4.3)$ & $3(16.7)$ & $2(20.0)$ & $0(0.0)$ & \\
\hline Smoking, $n(\%)$ & & & & & & & 0.031 \\
\hline Current & $16(45.7)$ & $9(24.3)$ & $20(42.6)$ & $4(22.2)$ & $6(60.0)$ & $16(72.7)$ & \\
\hline Never/stopped & $18(51.4)$ & $26(70.3)$ & $18(38.3)$ & $12(66.7)$ & $1(10.0)$ & $3(13.6)$ & \\
\hline Missing data & $1(2.9)$ & $2(5.4)$ & $9(19.2)$ & $2(11.1)$ & $3(30.0)$ & $3(13.6)$ & \\
\hline Presenting problem, $n(\%)$ & & & & & & & $<0.0001$ \\
\hline Cancer & $1(1.9)$ & $1(2.7)$ & $5(10.9)$ & $2(11.8)$ & $2(20.0)$ & $0(0.0)$ & \\
\hline $\mathrm{O} \& \mathrm{G}$ & $0(0.0)$ & $0(0.0)$ & $5(10.9)$ & $6(35.3)$ & $0(0.0)$ & $0(0.0)$ & \\
\hline Trauma & $16(47.1)$ & $18(48.7)$ & $16(34.8)$ & $3(17.7)$ & $3(30.0)$ & $5(22.7)$ & \\
\hline Appendicitis & $10(29.4)$ & $9(24.3)$ & $1(2.2)$ & $2(11.8)$ & $2(20.0)$ & $13(59.1)$ & \\
\hline Perforated peptic ulcer & $5(14.7)$ & $2(5.4)$ & $1(2.2)$ & $1(5.9)$ & $2(20.0)$ & $1(4.6)$ & \\
\hline Other & $2(5.9)$ & $7(18.9)$ & $28(59.6)$ & $4(22.2)$ & $0(0.0)$ & $3(13.6)$ & \\
\hline Missing data & $1(1.9)$ & $0(0.0)$ & $1(2.2)$ & $0(0.0)$ & $1(10.0)$ & $0(0.0)$ & \\
\hline \multicolumn{8}{|l|}{ Provider, $n(\%)$} \\
\hline Qualified surgeon & & & & & & & $<0.0001$ \\
\hline Present & $12(34.3)$ & $4(10.8)$ & $33(71.7)$ & $15(83.3)$ & $6(60.0)$ & $19(86.4)$ & \\
\hline Absent & $23(65.7)$ & $33(89.2)$ & $13(28.3)$ & $3(16.7)$ & $4(40.0)$ & $3(13.6)$ & \\
\hline Qualified anaesthetist & & & & & & & $<0.0001$ \\
\hline Present & $28(80.0)$ & $37(100.0)$ & $11(23.4)$ & $1(5.6)$ & $3(30.0)$ & $5(22.7)$ & \\
\hline Absent & $7(20.0)$ & $0(0.0)$ & $36(76.6)$ & $17(94.4)$ & $7(70.0)$ & $17(77.3)$ & \\
\hline \multicolumn{8}{|l|}{ Process, $n(\%)$} \\
\hline Delay to theatre $(\mathrm{h})$ & & & & & & & 0.003 \\
\hline$<6$ & $17(48.6)$ & $16(43.2)$ & $12(25.5)$ & $6(33.3)$ & $3(30.0)$ & $12(54.6)$ & \\
\hline $6-11$ & $6(17.1)$ & $4(10.8)$ & $7(14.9)$ & $2(11.1)$ & $4(40.0)$ & $7(31.8)$ & \\
\hline $13-23$ & $8(22.9)$ & $11(29.7)$ & $12(25.5)$ & $2(11.1)$ & $1(10.0)$ & $2(9.1)$ & \\
\hline $24-47$ & $2(5.7)$ & $3(8.1)$ & 7 (14.9) & $4(22.2)$ & $0(0.0)$ & $1(4.6)$ & \\
\hline$>47$ & $2(5.7)$ & $3(8.1)$ & $9(19.2)$ & $4(22.2)$ & $2(20.0)$ & $0(0.0)$ & \\
\hline
\end{tabular}


Table 1. (continued) Comparison of patient characteristics $(N=169)$ by participating hospital

\begin{tabular}{|c|c|c|c|c|c|c|c|}
\hline \multirow[b]{2}{*}{ Patient characteristics } & \multicolumn{6}{|c|}{ Hospital } & \multirow[b]{2}{*}{$p$-value } \\
\hline & 1 & 2 & 3 & 4 & 5 & 6 & \\
\hline CT performed preoperatively & & & & & & & $<0.0001$ \\
\hline Yes & $3(8.6)$ & $0(0.0)$ & $21(44.7)$ & $6(33.3)$ & $2(20.0)$ & $20(90.9)$ & \\
\hline No & $32(91.5)$ & $37(100.0)$ & $26(55.3)$ & $12(66.7)$ & $8(80.0)$ & $2(9.1)$ & \\
\hline Blood transfusion & & & & & & & 0.789 \\
\hline Yes & $7(20.0)$ & $9(24.3)$ & $13(27.7)$ & $4(22.2)$ & $4(40.0)$ & $4(18.2)$ & \\
\hline No & $28(80.0)$ & $28(75.7)$ & $34(72.3)$ & $14(77.8)$ & $6(60.0)$ & $18(81.8)$ & \\
\hline Resection performed & & & & & & & 0.941 \\
\hline Yes & $6(17.1)$ & $8(21.6)$ & $7(14.9)$ & $2(11.1)$ & $1(10.0)$ & $3(13.6)$ & \\
\hline No & $29(82.9)$ & $29(78.4)$ & $40(85.1)$ & $16(88.9)$ & $9(90.0)$ & $19(86.4)$ & \\
\hline Stoma sited & & & & & & & 0.038 \\
\hline Yes & $3(8.6)$ & $1(2.7)$ & $10(21.3)$ & $3(16.7)$ & $2(20.0)$ & $0(0.0)$ & \\
\hline No & $32(91.5)$ & $36(97.3)$ & $37(78.7)$ & $15(83.3)$ & $8(80.0)$ & $22(100.0)$ & \\
\hline Operation & & & & & & & $<0.0001$ \\
\hline Negative & $1(2.9)$ & $4(10.8)$ & $8(19.1)$ & $1(5.6)$ & $1(10.0)$ & $0(0.0)$ & \\
\hline Foregut & $11(31.4)$ & $6(16.2)$ & $3(6.4)$ & $3(16.7)$ & $3(30.0)$ & $2(9.1)$ & \\
\hline Midgut & $14(40.0)$ & $16(43.2)$ & $5(10.7)$ & $8(44.4)$ & $2(20.0)$ & $16(72.7)$ & \\
\hline Hindgut & $4(11.4)$ & $6(16.2)$ & $7(14.9)$ & $3(16.7)$ & $2(20.0)$ & $2(9.1)$ & \\
\hline Other & $5(14.7)$ & $5(13.5)$ & $24(51.1)$ & $3(16.7)$ & $2(20.0)$ & $2(9.1)$ & \\
\hline
\end{tabular}

performed $(p<0.0001)$. No patient received a CT scan preoperatively in hospital 2 , whereas $90.9 \%$ in hospital 6 received one. The negative laparotomy rate was as low as $0 \%$ in hospital 6 and as high as $19.1 \%$ in hospital 3. Completion rates for the information included in Table 1 were very high. Data were missing only for current smoking status in 20/169 (11.8\%) and presenting problem in $3 / 169(1.8 \%)$.

\section{Occurrence of endpoints}

The overall in-hospital mortality rate was 13.4\% (95\% confidence interval (CI) 8.78 19.98), the rate of major complications $17.5 \%$ (95\% CI $12.11-24.49)$, the rate of any complications 33.6\% (95\% CI 26.36 - 41.61), the rate of minor complications $15.4 \%$ (95\% CI $10.43-22.25)$ and the LOS $>14$ rate $16.8 \%$ (95\% CI 11.55 - 23.75). Unadjusted outcomes differed among the participating hospitals, as presented in Table 2. The unadjusted in-hospital mortality rate ranged from $6.4 \%$ to $40.0 \%(p=0.043)$, the major in-hospital mortality rate from $5.4 \%$ to $40.0 \%(p=0.002)$, the minor in-hospital complication rate from $0 \%$ to $27.7 \%(p=0.018)$ and the any in-hospital complication rate from $16.7 \%$ to $70.0 \%$ ( $p=0.002$ ). The unadjusted LOS $>14$ rate did not differ by hospital $(p=0.572)$. Completion rates for outcomes of interest in this study were adequate. Data on in-hospital mortality were missing in 4 cases (2.4\%), on in-hospital morbidity in $12(7.1 \%)$ and on LOS in $14(8.3 \%)$. The majority of these missing data came from the hospital 4 cohort, which had the most scanty follow-up of patients, only reporting complications on 11 cases (61.1\%) and LOS data on 15 (83.3\%). The results of the multivariate analysis show that hospital location of surgery was an independent risk factor for in-hospital mortality, minor complications and major complications after controlling for all the 5P (patient, provider, presenting problem and process) variables considered in Table 1 . The final logistic regression models predicting an adverse outcome of interest following emergency abdominal surgery are shown in Table 3. A patient undergoing emergency abdominal surgery at hospital 5 had a 76 -fold increased odds of in-hospital death (95\% CI 8.94 646.93) and a 58-fold increased odds of a major in-hospital complication (95\% CI 7.09 - 478.7) requiring critical care admission compared with the reference hospital, after controlling for the abovementioned confounders. Surgery at hospital 3 was associated with a 3 -fold increased odds of any in-hospital complication (95\% CI 5.01 - 458.85). Although a minor in-hospital complication was associated with a 10 -fold increased odds of LOS $>14$, the hospital site was not an independent risk factor for prolonged LOS. The logistic regression model developed for predicting in-hospital mortality following emergency abdominal surgery in the SA hospitals contributing to GlobalSurg-1 has an
ROC value of 0.8892 (95\% CI 0.789 - 0.9234 ) (Fig. 2).

\section{Discussion}

In comparing outcomes in emergency abdominal surgery in SA hospitals using the $5 \mathrm{P}$ framework, this study found that hospital site was an independent predictor of in-hospital mortality and morbidity. Overall trauma was the most common presenting problem in the study, although this varied between hospitals, as did the demographic characteristics of the patients and the characteristics of the treating surgeons and anesthetists. Hospital cohorts were quite different, particularly with regard to the process of care variables, where the variation in presence of qualified specialists and the use of CT scans was most striking. Interestingly, but perhaps unsurprisingly, the hospital with the most liberal use of CT scanning reported no negative laparotomies. These findings are consistent with and extend those from the limited number of previous reports looking at variation in surgical outcomes from noncardiac procedures performed in SA and internationally.

The European Surgical Outcomes Study (EuSOS) was conducted in 2011 with the primary objective of describing mortality rates and patterns of critical care resource use for patients undergoing non-cardiac surgery across 28 European nations. ${ }^{[2]}$ The results 
Table 2. Unadjusted outcome measures by participating hospital

\begin{tabular}{|c|c|c|c|c|c|c|c|}
\hline \multirow[b]{2}{*}{ Outcome } & \multicolumn{6}{|c|}{ Hospital } & \multirow[b]{2}{*}{$p$-value } \\
\hline & 1 & 2 & 3 & 4 & 5 & 6 & \\
\hline In-hospital mortality, $n(\%)$ & & & & & & & 0.043 \\
\hline Yes & $3(8.6)$ & $6(16.2)$ & $3(6.4)$ & $2(11.1)$ & $4(40.0)$ & $2(9.1)$ & \\
\hline No & $32(91.4)$ & $30(81.1)$ & $43(91.5)$ & $15(83.3)$ & $5(50.0)$ & $20(90.9)$ & \\
\hline Missing data & $0(0.0)$ & $1(2.7)$ & $1(2.1)$ & $1(5.6)$ & $1(10.0)$ & $0(0.0)$ & \\
\hline Major complication, $n(\%)$ & & & & & & & 0.002 \\
\hline Yes & $2(5.7)$ & $2(5.4)$ & $14(29.8)$ & $3(16.7)$ & $4(40.0)$ & $2(9.1)$ & \\
\hline No & $33(94.3)$ & $32(86.5)$ & $32(68.1)$ & $8(44.4$ & $5(50.0)$ & $20(90.9)$ & \\
\hline Missing data & $0(0.0)$ & $3(8.1)$ & $1(2.1)$ & $7(38.9)$ & $1(10.0)$ & $0(0.0)$ & \\
\hline Minor complication, $n(\%)$ & & & & & & & 0.018 \\
\hline Yes & $5(14.3)$ & $1(2.7)$ & $13(27.7)$ & $0(0.0)$ & $2(20.0 .0)$ & $2(9.1)$ & \\
\hline No & $30(85.7)$ & $33(89.2)$ & $33(70.2)$ & $11(61.1)$ & $7(70.0)$ & $20(90.9)$ & \\
\hline Missing data & $0(0.0)$ & $3(8.1)$ & $1(2.1)$ & $7(38.9)$ & $1(10.0)$ & $0(0.0$ & \\
\hline Any complication, $n(\%)$ & & & & & & & 0.002 \\
\hline Yes & $8(22.9)$ & $7(18.9)$ & $22(46.8)$ & $3(16.7)$ & $7(70.0)$ & $4(18.2)$ & \\
\hline No & $27(77)$. & $28(75.7)$ & $24(51.1)$ & $8(44.4)$ & $2(20.0)$ & $18(18.8)$ & \\
\hline Missing data & $0(0.0)$ & $2(5.4)$ & $1(2.1)$ & $7(38.9)$ & $1(10.0)$ & $0(0.0)$ & \\
\hline LOS $>14, n(\%)$ & & & & & & & 0.572 \\
\hline Yes & $4(11.4)$ & $3(8.1)$ & $10(21.3)$ & $3(16.7)$ & $3(30.0)$ & $3(13.6)$ & \\
\hline No & $30(85.7)$ & $26(70.3)$ & $36(76.6)$ & $12(66.7)$ & $7(70.0)$ & $18(18.8)$ & \\
\hline Missing data & $1(2.9)$ & $8(21.6)$ & $1(2.1)$ & $3(16.7)$ & $0(0.0)$ & $1(4.6)$ & \\
\hline
\end{tabular}

showed that mortality rates varied 20 -fold between countries (from $1.2 \%$ for Iceland to $21.5 \%$ for Latvia), despite adjustment for confounding variables. This meant that, after adjustment for variations in perioperative factors, a patient was up to seven times more likely to die postoperatively simply because of the hospital or country location of the surgery.

Following the findings of this landmark study in the emerging field of surgical outcomes research, EuSOS provided the rationale for Biccard et al. ${ }^{[6]}$ to conduct a similar 7-day cohort study known as the South African Surgical Outcomes Study (SASOS) during 2014 in SA. The primary outcome was in-hospital mortality. In SASOS, crude in-hospital mortality rates were 123/3 927 (3.1\%, 95\% CI 2.6 - 3.7). Urgent or emergency surgery occurred in $2120 / 3915$ (54.2\%), with a population-attributable risk for mortality of 25.5\% (95\% CI 5.1 - 55.8). SASOS concluded that most patients in SA undergo urgent and emergency surgery, which is strongly associated with mortality and unplanned critical care admissions. Based on the estimates of surgical volumes in SA by Weiser et al. ${ }^{[7]}$ the population statistics of SA for $2013^{[8]}$ and the SASOS data, ${ }^{[6]}$ the estimated mortality rate for adults ( $\geq 20$ years of age) undergoing surgery in SA is between 76 and $128 / 100000$, which is equivalent to $7.2 \%$ and $12.1 \%$, respectively,

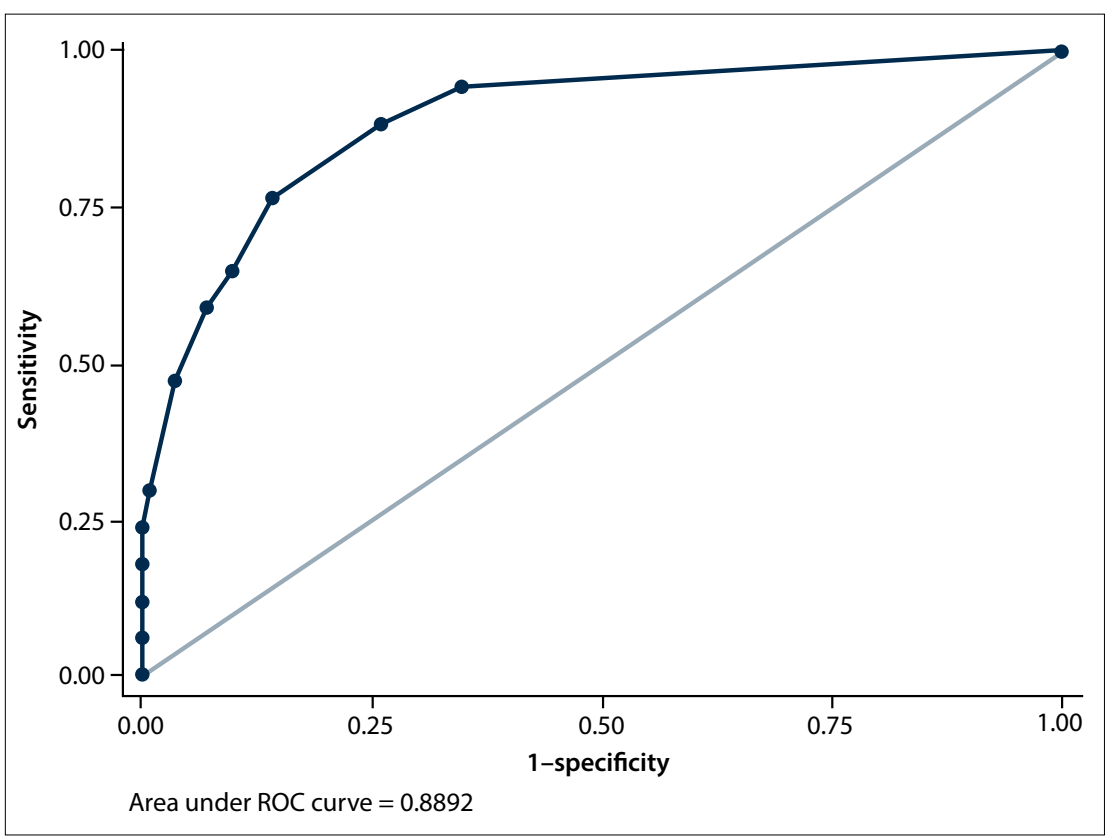

Fig. 2. Area under the ROC for the model predicting in-hospital mortality following emergency intraperitoneal surgery in GlobalSurg SA.

of all deaths in SA. These estimates provide substantial support for more in-depth analyses of the quality of surgical care provided in SA.

With no current national dataset collating surgical outcomes of non-cardiac surgery in SA, targeting emergency laparotomies is a logical starting point. Resource-intensive national quality improvement (QI) programmes such as the American College of Surgeons National Surgical QI Essentials Program that only report specialty-specific outcomes (general surgery, orthopaedic 
Table 3. Logistic regression models built to predict in-hospital complications following emergency intraperitoneal surgery in GlobalSurg SA

\begin{tabular}{|c|c|c|c|}
\hline & Odds ratio & 95\% CI & $p$-value \\
\hline \multicolumn{4}{|l|}{ In-hospital mortality } \\
\hline Hospital 5 & 76.05 & $8.94-646.93$ & $<0.0001$ \\
\hline Age category elderly & 30.98 & $3.18-302.04$ & 0.003 \\
\hline ASA category 3 & 9.79 & $1.69-56.79$ & 0.011 \\
\hline ASA category 4 & 23.64 & $3.09-180.84$ & 0.002 \\
\hline Any resection & 4.46 & $0.99-20.02$ & 0.051 \\
\hline \multicolumn{4}{|l|}{ Major in-hospital complication } \\
\hline Hospital 5 & 58.24 & $7.09-478.7$ & $<0.001$ \\
\hline Age category elderly & 33.32 & $4.95-224.16$ & $<0.001$ \\
\hline ASA category 3 & 8.25 & $1.98-34.33$ & 0.004 \\
\hline ASA category 4 & 12.95 & $1.97-85.01$ & 0.008 \\
\hline Diagnosis of perforated peptic ulcer & 5.55 & $1.71-26.31$ & 0.031 \\
\hline CT scan not performed & 4.28 & $1.02-17.89$ & 0.047 \\
\hline \multicolumn{4}{|l|}{ Minor complication } \\
\hline Hospital 3 & 3.3 & $1.27-8.59$ & 0.015 \\
\hline \multicolumn{4}{|l|}{ Any complication } \\
\hline Hospital 5 & 47.92 & $5.01-458.85$ & 0.001 \\
\hline Hospital 3 & 3.08 & $1.27-7.49$ & 0.013 \\
\hline Age category elderly & 7.41 & $1.46-37.67$ & 0.016 \\
\hline ASA 3 & 3.13 & $1.09-9.02$ & 0.035 \\
\hline ASA 4 & 3.46 & $0.81-14.72$ & 0.043 \\
\hline Delay $>47 \mathrm{~h}$ & 2.84 & $0.96-8.39$ & 0.059 \\
\hline \multicolumn{4}{|l|}{ LOS $>14$} \\
\hline Presence of minor complication & 10.36 & $2.71-39.54$ & 0.001 \\
\hline Blood transfusion & 7.03 & $1.99-24.75$ & 0.002 \\
\hline Diagnosis of cancer & 19.91 & $2.28-173.43$ & 0.007 \\
\hline Diagnosis of perforated peptic ulcer & 8.64 & $0.69-108.11$ & 0.04 \\
\hline Delay $24-47 \mathrm{~h}$ & 4.19 & $1.04-16.85$ & 0.043 \\
\hline
\end{tabular}

surgery, etc.) may not be sufficiently granular for targeting specific procedures or subspecialty areas for improvement. They may even be falsely reassuring and result in missed opportunities for improvement. ${ }^{[9]}$ For example, a hospital's poor performance in colorectal surgery may be masked by better-than-average outcomes in trauma surgery, or vice versa. Procedure-specific performance measures would alleviate such problems. Focusing on procedure-specific outcomes assessment in a resource-limited setting would also reduce the amount of information needed for risk adjustment. ${ }^{[10]}$ In LMICs, effort needs to be focused on receiving the greatest return on investment of time and resources. Emergency exploratory laparotomy is both common and high risk. It has the added benefit that various general surgery subspecialties and general surgeons, as well as gynaecologists, perform it.
The published protocol for GlobalSurg-1 specifically excluded analyses on individual hospitals and surgeons. Although we have focused analyses on individual hospitals as comparators, this work is still in keeping with Donabedian's philosophies of 'system' measures and not 'human' measures. ${ }^{[1]}$ Hospitals function as complex systems, and pursuing further work in this field under the novel structure, process and outcomes model will continue to destigmatise the individual as a target of 'blame' for unfavourable outcomes and emphasise a broader understanding, in that QI requires awareness that the system is ultimately what contributes to most of the variation seen in outcomes. ${ }^{[12]}$ This will hold true particularly in the SA public sector, and we should pursue further research in this area without hesitation.

These data must be interpreted in the context of the study design. Reliable surgical outcomes data in the public sector of SA are not routinely measured, and any findings in this field are exploratory and preliminary. The effective sample size of 169 shared by six hospitals is particularly small considering that the primary outcome occurred relatively infrequently. Our estimates are therefore not precise, as reflected by the very large CIs. However, despite these imprecise estimates, there is consistency in this emerging field in the literature, and this makes us confident about the following closing comments:

- Non-cardiac surgery performed in hospitals across the world is subject to performance variation.

- Emerging web-based mobile-health technologies, such as REDCap, make rapid prospective database development and risk-adjusted benchmarking feasible, even in a resource-limited environment.

- Complications following major non-cardiac surgery, and particularly emergency surgery, are a common cause of death in SA and further work in this field is encouraged.

- The emergency exploratory laparotomy is a sensible procedure to target for a surgical QI initiative.

- Collaboration is key. ${ }^{[11]}$

\section{Conclusion}

The hospital is an independent risk factor for risk-adjusted adverse outcomes following emergency intraperitoneal surgery in SA.

\section{References}

1. Meara JG, Leather AJ, Hagander L, et al. Global Surgery 2030: Evidence and solutions for achieving health, welfare, and economic development. Lancet 2015;386(9993):569-624. [http://dx.doi.org/10.1016/S0140-6736(15)60160-X]

2. Pearse RM, Rhodes A, Moreno R, et al. EuSOS: European Surgical Outcomes Study. Eur J Anaesthesiol 2011;28(6):454456. [http://dx.doi.org/10.1097/EJA.0b013e328344907b]

3. Haynes AB, Regenbogen SE, Weiser TG, et al. Surgical outcome measurement for a global patient population: Validation of the measurement for a global patient population: Validation of the
Surgical Apgar Score in 8 countries. Surgery 2011;149(4):519524. [http://dx.doi.org/10.1016/j.surg.2010.10.019]

4. Bhangu A. Determining universal processes related to best Bhangu A. Determining universal processes related to best outcome in emergency abdominal surgery: A multicentre,
international, prospective cohort study. BMJ 2014;4(10):e006239. international, prospective cohort study. BM] 2014;4(10):
[http://dx.doi.org/10.1136/bmjopen-2014-006239]

5. Project REDCap. 2015. http://www.project-redcap.org/ (accessed 24 June 2015).

6. Biccard BM, Madiba TE. The South African Surgical Outcomes Study: A 7-day prospective observational cohort study. S Afr Med J 2015;105(6):465-475. [http://dx.doi.org/10.7196/SAMJ.9435]

7. Weiser TG, Regenbogen SE, Thompson KD, et al. An estimation of the global volume of surgery: A modelling strategy based on available data. Lancet 2008;372(9633):139-144. [http://dx.doi. org/10.1016/S0140-6736(08)60878-8]

8. Statistics South Africa. 2013. http://www.statssa.gov.za/ (accessed 24 June 2015).

9. Birkmeyer JD, Shahian DM, Dimick JB, et al. Blueprint for a new American College of Surgeons: National Surgical Quality new American College of Surgeons: National Surgical Quality
Improvement Program. J Am Coll Surg 2008:207(5):777-782. Improvement Program. J Am Coll Surg 2008;207(5
[http://dx.doi.org/10.1016/j.jamcollsurg.2008.07.018]

10. Andterson JE, Rose J, Noorbakhsh A, et al. An efficient risk adjustment model to predict inpatient adverse events after surgery. World J Surg 2014;38(8):1954-1960. [http://dx.doi.org/10.1007/s00268-014-2490-6] 11. Donabedian A. Evaluating the quality of medical care. Milbank Q 2005;83(4):691-729. [http://dx.doi.org/10.1111/j.1468-0009.2005.00397.x] 12. Spence RT, Panieri E, Rayne SL, et al. Strengthening surgical research capacity in sub-Saharan Africa: Collaboration is key. S Afr Med J 2016;106(2):xxxx. [http://dx.doi.org/10.7196/ SAMJ.2016.v106i2.10182]

Accepted 25 October 2015. 